\title{
CREACIÓN DE UNA PLANTILLA DE REDACCIÓN DE CONTRATOS DE COMPRAVENTA DE VIVIENDAS. UN ESTUDIO APLICADO A IRLANDA
}

\author{
Implementing a writing template for housing purchase \\ and sale agreements. A study applied to Ireland
}

\author{
Lorena Arce Romeral \\ Universidad de Valladolid (España) \\ Míriam Seghiri \\ Universidad de Málaga (España)
}

De acuerdo con el Instituto Nacional de Estadística (INE, 2018), actualmente 9.653 españoles residen en Irlanda, muchos de los cuales no sería de extrañar que acaben estableciéndose y adquiriendo su propia vivienda. Por su parte, según datos de la Organización Mundial del Turismo (OMT), España fue el segundo país más visitado en el 2018 y el turismo irlandés representó el $25 \%$ del total, de los que 15.119 decidieron afincarse en el país (INE, 2018). Estas tendencias en el sector turístico provocan un auge en el sector inmobiliario residencial entre España e Irlanda, lo que a su vez genera una creciente demanda de traducción al inglés de contratos de compraventa. Dada su estructura recurrente y estereotipada, el objetivo del estudio es la implementación de una plantilla de contratos de compraventa de viviendas que semiautomatice el proceso de redacción en inglés (variedad irlandesa). Para ello, puesto que los corpus lingüísticos constituyen fuentes muy valiosas de información en cuanto que macro- y microfuente documental (Corpas, 2008), nos basaremos en la gestión de un corpus representativo y de calidad de contratos de compraventa de viviendas en inglés (variedad irlandesa).

\section{Palabras clave}

Traducción especializada, contratos, compraventa, vivienda, lingüística de corpus
According to the Spanish National Institute of Statistics, 9,653 Spanish people currently reside in Ireland, many of whom end up settling and acquiring their own homes. Moreover, according to data from the World Tourism Organization (UNWTO), Spain was the second most visited country in 2018, with Irish tourism representing $25 \%$ of the total, of which 15,119 decided to settle in the country. These trends in the tourism sector are causing a boom in residential real estate between Spain and Ireland, which in turn is generating a growing demand for the translation of housing purchase and sale agreements into English. Given its recurrent and stereotyped structure, the aim of the study is to implement a template for housing purchase and sale agreement capable to semi-automate the process of writing in English (Irish variety). To this end, and given that linguistic corpora constitute very valuable sources of information in terms of macroand microdocumentary information (Corpas, 2008), the work will be based on the management of a representative and quality corpus of housing purchase and sale agreements in English (Irish variety).

Keywords

Specialized translation, agreements, purchase and sale, housing, corpus linguistics 


\section{Introducción}

Según datos dados a conocer por la Organización Mundial del Turismo (OMT), en el año 2018 España ocupó la segunda posición turística mundial. Así, nuestro país ha logrado batir su propio récord con un total de casi 82 millones de turistas extranjeros, lo que supone el mayor crecimiento turístico desde al año 2010. Por su parte, según el I. Informe prospectivo trimestral del turismo, España ocupa un papel destacado y popular en el turismo irlandés, pues durante el año 2018 fue el primer destino vacacional para los irlandeses, tanto en número de pernoctaciones ( $25 \%$ del total) como en gasto turístico (22 \% del total). De hecho, el turismo estival español ha crecido principalmente gracias a las llegadas procedentes de Reino Unido, Irlanda y Rusia (OMT, 2018), donde los primeros son los que más visitan nuestro país, seguidos por los ciudadanos irlandeses con un $36,5 \%$ del total. Por comunidades autónomas, según la Estadística de movimientos turísticos en fronteras (FRONTUR) del INE correspondiente al año 2018, el destino preferido fue las islas Baleares, con un 28,8\%; porcentaje muy cercano a Cataluña, con un $24,5 \%$; y Andalucía, con un 13,7 \%. Dichas tendencias han provocado, a su vez, un aumento considerable de población residente foránea en nuestro país. Así, según el último Censo de población y viviendas realizado (del año 2011), el porcentaje de población extranjera en España se sitúa entre el 15 y el $20 \%$ del total. Esto se traduce en más de cinco millones de personas, lo que ha supuesto un crecimiento del $234,1 \%$ en diez años (de acuerdo con los datos registrados por el anterior Censo de población y viviendas, del año 2001). Concretamente, según el INE, en 2019 vivían en nuestro país 15.119 irlandeses. En efecto, no es de extrañar que el señalado crecimiento de población provoque, a su vez, un aumento de la demanda de turismo residencial para responder a los turistas que se concentran durante el período estival, pero, principalmente, a los que desean adquirir una vivienda. Así lo recoge el periódico El Confidencial al afirmar: "Los extranjeros compran veinte de cada cien viviendas que se venden en España». De hecho, de acuerdo con el periódico El País, la Comunidad Valenciana sigue liderando el número de transacciones de compraventa inmobiliaria con 15.613 operaciones, seguido de Andalucía con 9.737 y de Cataluña con 7.570. Por nacionalidad, los irlandeses fueron los que más crecieron, con un $24,7 \%$.

En Irlanda, por su parte, la llegada de turistas españoles al país aumentó en el año 2018 un $12 \%$ (esto hace un total de 445.000 turistas), de acuerdo con los datos aportados por la oficina de turismo de dicho país en España. Asimismo, las cifras del Ministerio de Trabajo, Migraciones y Seguridad Social de España reflejan cantidades interesantes sobre la emigración española hacia Irlanda, sobre todo de jóvenes cualificados que desean mejorar sus conocimientos lingüísticos en inglés. Así, según datos del $I N E$, el número de españoles residentes actualmente en Irlanda es de 9.653, muchos de los cuales seguramente acabarán asentándose y adquiriendo su propia vivienda.

En definitiva, las cifras expuestas reflejan todo un negocio en auge, a nivel nacional e internacional, de compraventa de viviendas $y$, concretamente, existe una indiscutible demanda por parte de los españoles en cuanto a la redacción de contratos de compraventa de viviendas en inglés en su variedad irlandesa. Este hecho, unido al carácter estereotipado y recurrente que caracteriza el discurso jurídico de los contratos de compraventa de viviendas, nos llevará a crear, en los siguientes apartados, una plantilla que pueda semiautomatizar el proceso de redacción en inglés, en su variedad irlandesa, de contratos de compraventa de viviendas. El recurso ideal a partir del cual crearemos la mencionada plantilla será un corpus que puede definirse, en palabras de Sinclair (1991, p. 171), como «[...] una colección de textos de ocurrencias de lenguaje natural, escogidos para caracterizar un estado o una variedad de lengua», pues se configura como "ejemplos de esta lengua» (EAGLES, 1996, p. 4). Para ello, nos proponemos la compilación de un corpus virtual, fiable y de calidad compuesto por contratos de compraventa de viviendas en inglés en su variedad irlandesa. Seguidamente, puesto que toda traducción jurídica implica una comparación entre ordenamientos jurídicos, se identificará la legislación relativa a la compraventa de viviendas en Irlanda. Por último, se procederá a la del corpus compilado para generar una plantilla de redacción semiautomática de contratos de compraventa de viviendas conforme a la legislación y usos propios irlandeses.

\section{Legislación aplicable a la compraventa de viviendas en Irlanda}

La Constitución de Irlanda aborda de manera sucinta en el apartado Private Property -y más concretamente en el artículo 43 en sus diferentes apartados- el derecho a la propiedad privada, así como sus garantías constitucionales. Sin embargo, no regula de forma sustantiva la compraventa de viviendas.

Las continuas agitaciones por la tierra durante las décadas de los ochenta y los noventa del siglo XIX culminaron con la aprobación de las Land Acts (Ireland). Con ellas, se aprobaron una serie de medidas para abordar la cuestión de los contratos de arrendamiento y la propiedad campesina de la tierra. Destacan, en primer lugar, las leyes aprobadas por 
el Gobierno del Reino Unido entre 1870 y 1909. Estas fueron la Purchase of Land (Ireland) Act 1885, enmendada por la Land Purchase Act, 1887; así como la Land (Purchase) Act 1903, la Labourers (Ireland) Act 1906 y, finalmente, la Irish Land (Provision for Sailors and Soldiers) Act 1919. Después de 1922, una vez tuvo lugar la aceptación del Tratado Anglo-Irlandés, por medio del cual se estableció el Estado Libre de Irlanda, se aprobó la Land Act, 1923, que viene a modificar las anteriormente mencionadas.

A título particular, las Land Acts fueron una serie de medidas para abordar la cuestión de la propiedad campesina de la tierra en Irlanda entre los siglos XIX y XX. Las continuas agitaciones por la tierra durante las décadas de los ochenta y los noventa del siglo XIX culminaron, en primer lugar, con la aprobación de la Purchase of Land (Ireland) Act 1885, enmendada por la Land Purchase Act, 1887. Más tarde, se aprobó la Land Act, 1923, así como la Land (Purchase) Act 1903, por la que se modifican materias relativas a la ocupación, seguidas de la Labourers (Ireland) Act 1906 y la Irish Land (Provision for Sailors and Soldiers) Act 1919.

De más reciente aprobación, destaca la Land and Conveyancing Law Reform Act 2009, que modernizó la adquisición de la propiedad, modificada, asimismo, por las Land and Conveyancing Law Reform Act 2013 y 2019. En materia de registro de títulos destaca la Registration of Title Act 1964, que ha sido enmendada por la Registration of Deeds and Title Act 2006 a fin de facilitar la creciente informatización de los sistemas de registro de la propiedad y el derecho de sucesiones (Succession Act 1965). Estas, a su vez, han sido reformadas por las Land Registration (Fees) Order 1966, 1974, 1978, 1980, 1981, 1983, 1987, 1991 y 1999 y la Land Registration Rules 1975, 1977, 1986, 2000, 2005, 2006, 2007, 2009, 2011, 2012 y 2013. A estas leyes se suma una amplia protección legal de los bienes familiares con la Family Home Protection Act 1976.

La Property Registration Authorithy (PRA) es el organismo estatal responsable del registro tanto de las transacciones inmobiliarias como de la propiedad de la tierra en Irlanda. Se trata de un órgano estatuario creado el 4 de noviembre de 2006 en virtud de las disposiciones de la Title Act 2006. Las principales funciones de la PRA son aquellas de gestionar y controlar el Land Registry (en español, Registro de la Propiedad) y el Registry of Deeds (el Registro de Escrituras) ${ }^{1}$. En lo que a la redacción del contrato de compraventa de refiere, fue en al año 1976

${ }^{1}$ El Land Registry (Registro de la Propiedad) fue creado en 1892 $y$, una vez ha sido inscrita la propiedad en dicho registro, esta se debe registrar en el Registry of Deeds (Registro de Escrituras). cuando la institución Law Society of Ireland ${ }^{2}$ introdujo por primera vez un modelo estándar de contrato de compraventa de viviendas, a fin de suprimir la reducción individual de dichos contratos y garantizar un equilibro justo entre el comprador y el vendedor. Así, aunque hasta la fecha la Law Society of Ireland ha modificado dicho modelo, comprende una serie de aspectos esenciales abordados en el apartado 3.2.1, dedicado a la estructura de los contratos de compraventa de viviendas en inglés en su variedad irlandesa.

\section{Diseño de plantillas de redacción en inglés (variedad irlandesa) de contratos de compraventa de viviendas basadas en corpus}

En las siguientes páginas se ilustra el proceso de creación de plantillas de redacción en inglés, en su variedad de Irlanda ${ }^{3}$, de contratos de compraventa de viviendas basada en la explotación de un corpus virtual creado para tal fin, así como una breve descripción del protocolo seguido para su compilación.

\subsection{Compilación de un corpus virtual de contratos de compraventa de viviendas en inglés (variedad irlandesa)}

Para que un determinado conjunto de textos pueda considerarse un corpus, debe ser representativo, es decir, debe representar, tal y como afirma Corpas (2001, p. 156), «[...] el estado de una lengua o de la sección de lengua específicamente seleccionada». Dicha representatividad, tal y como apunta Seghiri (2006, 2011 o 2015), puede entenderse desde dos puntos de vista: el cualitativo y el cuantitativo. Desde el punto de vista de la calidad, un corpus, según Seghiri (2006, p. 256), "será representativo si está correctamente diseñado y, por tanto, se ha realizado una correcta selección de documentos que lo integran». De esta manera, para garantizar la representatividad cualitativa del corpus es necesario, en primer lugar, establecer unos parámetros claros de diseño $y$, en segundo lugar, una compilación protocolizada. Por último, para asegurar la representatividad desde el punto de vista cuantitativo recurriremos al programa informático ReCor, diseñado para tal fin.

\footnotetext{
${ }^{2}$ Law Sociedy of Ireland es un órgano educativo, representativo y regulador de la profesión de abogado en la República de Irlanda, de acuerdo con Solicitors Acts, 1954-2015.

${ }^{3}$ Un proceso similar se ha seguido para la compilación de un corpus, así como plantillas de contratos de compraventa de viviendas en español (variedades peninsular y argentina) e inglés (variedades británica y estadounidense). Para más información, véase Arce y Seghiri (2018 y 2019 y 2020 en prensa).
} 


\subsubsection{Determinación de la representatividad cualitativa}

En primer lugar, nos proponemos establecer unos parámetros claros para el diseño de nuestro corpus de contratos de compraventa de viviendas. Así, el corpus que pretendemos compilar será virtual (integrado únicamente por textos descargados de la red Internet), comparable (se conforma por contratos originales de viviendas y no traducciones) y, por ende, especializado (pues es de contenido jurídico) y completo (dado que lo componen contratos de compraventa de viviendas íntegros). Asimismo, será un corpus monolingüe (en inglés), delimitado diatópicamente a contratos de compraventa de viviendas redactados en Irlanda. Finalmente, el corpus será documentado, ya que se recogerá la fuente del texto descargado de la red, y plano, esto es, no etiquetado. Determinados los criterios de diseño iniciales, el siguiente paso consiste en establecer el protocolo de compilación del corpus. Concretamente, nos hemos basado en la metodología de compilación propuesta por Seghiri (2006, 2012, 2017a y 2017b), integrada en cuatro fases, a saber, localización de textos, descarga, formato y almacenamiento.

En primer lugar, para abordar la fase I (localización de los textos), puesto que se trata de un corpus virtual, estará integrado únicamente por textos descargados de Internet. De esta forma, para la localización de los contratos nos hemos valido principalmente de dos tipos de búsquedas en red: de un lado, la búsqueda institucional y, de otro, la búsqueda avanzada en buscadores (como Google) a través de descriptores y ecuaciones booleanas. Así, la búsqueda institucional es aquella realizada a través de portales de instituciones, asociaciones u organismos oficiales. Como botón de muestra de este tipo de búsqueda, se puede mencionar la consulta a la plataforma vLex Ireland, una de las mayores colecciones de información jurídica a nivel mundial, que permite acceder a diversos formularios y contratos disponibles a texto completo, con actualización diaria, redactados en el ordenamiento jurídico irlandés (cfr. Figura 1).

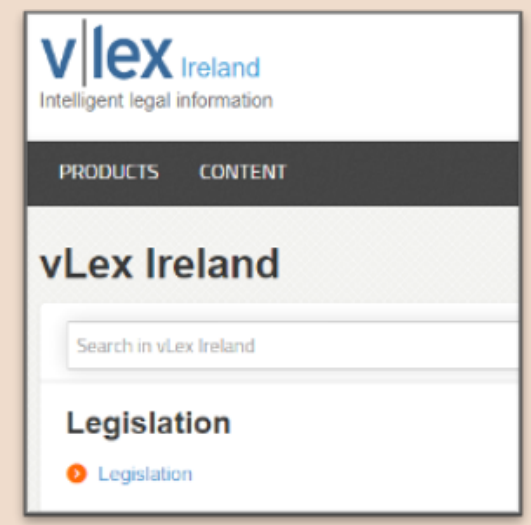

Figura 1. Búsqueda institucional a través de VLex Ireland.
En segundo lugar, la búsqueda avanzada en Google permite, entre otras funciones, delimitar el idioma (inglés) y la zona geográfica (Irlanda). Asimismo, es posible aplicar descriptores y ecuaciones de búsqueda, que se recogen en la siguiente tabla (cfr. Tabla 1), a fin de filtrar todo lo posible el ruido documental.

\begin{tabular}{|c|c|c|c|c|c|}
\hline $\begin{array}{c}\text { Busca- } \\
\text { dor }\end{array}$ & Lengua & $\begin{array}{c}\text { Varie- } \\
\text { dad }\end{array}$ & $\begin{array}{c}\text { Tipo } \\
\text { textual }\end{array}$ & $\begin{array}{c}\text { Descrip- } \\
\text { tores }\end{array}$ & $\begin{array}{c}\text { Ecuacio- } \\
\text { nes de } \\
\text { búsque- } \\
\text { da }\end{array}$ \\
\hline Google & Inglés & Irlandés & $\begin{array}{c}\text { Purcha- } \\
\text { se and } \\
\text { sale } \\
\text { agree- } \\
\text { ment }\end{array}$ & $\begin{array}{c}\text { Purcha- } \\
\text { se, sale, } \\
\text { agree- } \\
\text { ment, } \\
\text { house, } \\
\text { dwe- } \\
\text { Iling } \\
\text { sing pur- } \\
\text { chase } \\
\text { and sale } \\
\text { agree- } \\
\text { ment" } \\
\text { AND } \\
\text { dwelling } \\
\text { OR } \\
\text { house }\end{array}$ \\
\hline
\end{tabular}

Tabla 1. Descriptores y ecuaciones de búsqueda para la búsqueda avanzada.

Una vez hemos localizado contratos de compraventa de viviendas redactados en Irlanda, procederemos a su descarga. Este proceso puede realizarse de forma manual (cfr. Figura 2).

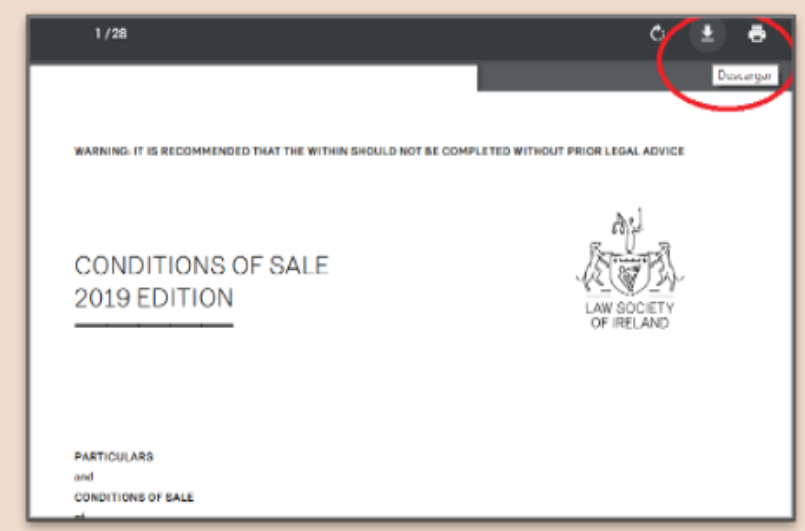

Figura 2. Descarga manual de un contrato de compraventa en formato PDF.

Sin embargo, también puede automatizarse la descarga de un grupo de contratos a través de determinadas aplicaciones que permiten la descarga «en lotes», como GNUWget o GetBot (cfr. Figura 3).

Dada la variedad de formatos en los que podemos encontrar los textos compilados, unido a que la mayoría de los programas de gestión de corpus únicamente trabaja con documentos en formato ASCII o texto plano (.txt), se hace necesario iniciar 


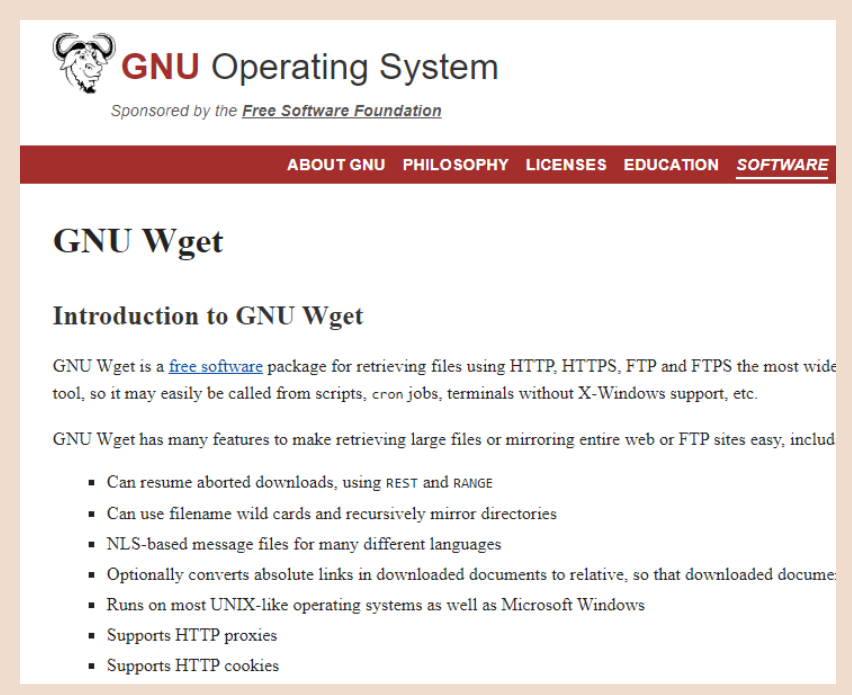

Figura 3. Descarga automática de documentos con GNU Wget.

un proceso de conversión de formato de los contratos. Podemos llevar a cabo esta tarea copiando el contenido del documento original y pegándolo, seguidamente, en un documento de texto plano, tal y como propone Seghiri (2012, p. 376): «The conversión from any format to plain is as easy as to copy and paste it into a plain text document (.txt)» (cfr. Figura 4). Esto puede hacerse prácticamente en todos los casos, siempre y cuando los documentos no se encuentren encriptados.

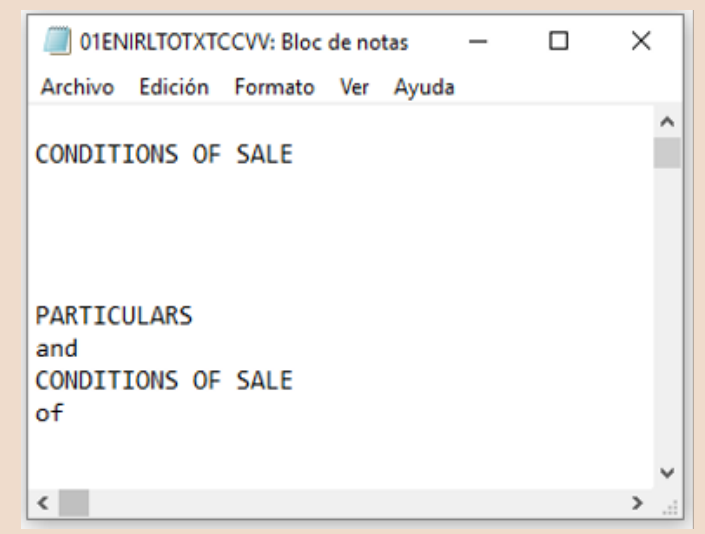

Figura 4. Conversión a formato ASCIl (texto plano .txt).

Si el documento original se encuentra encriptado (muy frecuente en el caso del formato .pdf), es necesario recurrir a aplicaciones informáticas específicas, tales como Pdftoword o Freepdfconvert, por ejemplo (cfr. Figura 5).

La última fase (almacenamiento) del proceso de compilación consiste en clasificar en carpetas y subcarpetas los documentos resultantes. Para ello, es necesario diseñar una codificación que permita localizar los textos rápidamente, así como una futu-

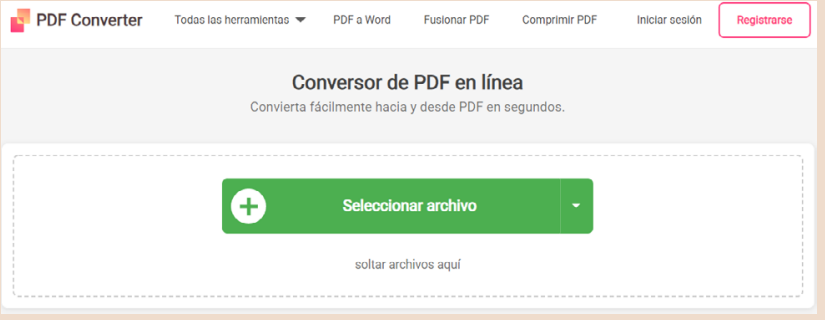

Figura 5. Conversión de PDF a formato ASCIl con Freepdfconvert.

ra ampliación del corpus. De esta forma, en primer lugar hemos creado una carpeta llamada CCVV (contratos de compraventa de viviendas) y dentro de ella encontramos una carpeta denominada EN (inglés). Seguidamente, crearemos una nueva subcarpeta, a saber, IRL (documentos en inglés, en la variedad irlandesa). Dentro de esta subcarpeta se creará otra llamada TO (para almacenar los textos originales). En el interior de esta, se albergarán dos, a saber, FO (para conservar los contratos en formato original) y TXT (donde se guardarán los contratos convertidos a texto plano). En consecuencia, si atendemos a esta codificación, un documento que cuente con la codificación 01ENIRLTOTXTCCVV significará que estamos ante el documento número uno del corpus (01), redactado en inglés (EN), en la variedad irlandesa (IRL), que es un texto original (TO) y que, además, se ha convertido a texto plano (TXT) y contiene un contrato de compraventa de viviendas (CCVV). En la Tabla 2 se ejemplifica el sistema de almacenamiento y codificación seguidos.

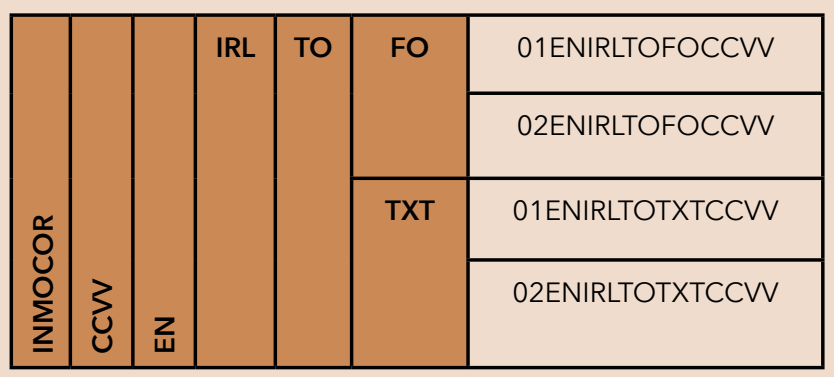

Tabla 2. Sistema de almacenamiento y codificación del corpus.

Gracias al establecimiento de los criterios de diseño y el protocolo de compilación establecidos, hemos obtenido un corpus representativo desde el punto cualitativo que es virtual (compuesto exclusivamente por textos descargados de Internet), comparable (integrado por contratos originales), monolingüe (inglés), pero delimitado diatópicamente a contratos de compraventa de viviendas redactados en Irlanda. Además, se trata de un corpus jurídico y, por tanto, especializado, documentado y plano, 
compuesto por 10 contratos (y 48.070 palabras o tokens) ${ }^{4}$ de contratos de compraventa.

\subsubsection{Determinación de la representatividad cuantitativa}

Una vez hemos asegurado la representatividad cualitativa del corpus compilado, gracias a los criterios de diseño y al protocolo de compilación (en cuatro fases) aplicados, el siguiente paso consiste en comprobar si, con la totalidad de documentos que integra el corpus, hemos obtenido una muestra representativa desde el punto de vista de la cantidad. De esta forma, se trata de comprobar si se ha cubierto la terminología básica empleada en este género textual (los contratos de compraventa de viviendas) para cada variedad diatópica objeto de estudio, a saber, el inglés irlandés. Para ello, nos hemos valido de la aplicación informática ReCor ${ }^{5}$, que permite estimar la representatividad de los corpus en función de su tamaño a través del algoritmo N-Cor (cfr. Seghiri, 2006, 2011 y 2015). En este caso, vamos a utilizar la versión 2.0 de $\operatorname{ReCor}^{6}$ (cfr. Figura 6).

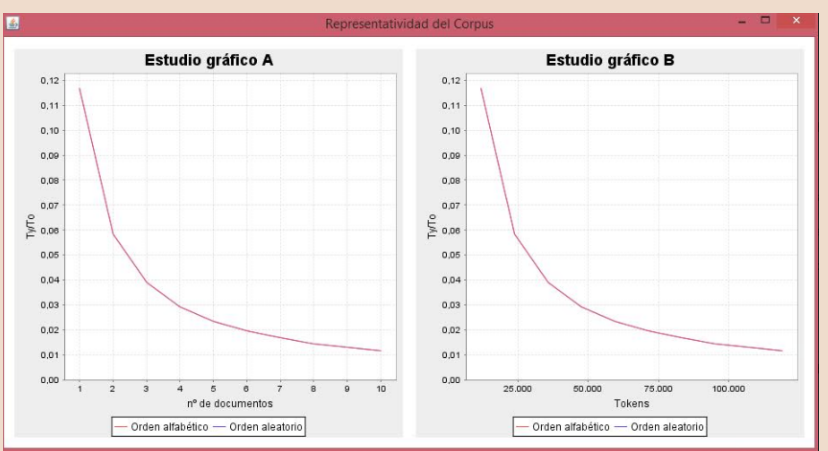

Figura 6. Determinación de la representatividad del corpus de contratos de compraventa en inglés (variedad irlandesa) para 1-grama con ReCor.

De la gráfica anterior (cfr. Figura 6) se deduce que la línea que toma los documentos en orden alfabético (en color rojo) coincide desde el principio exactamente con la que analiza los textos en orden

\footnotetext{
${ }^{4}$ Para contar el número de palabras, hemos empleado la herramienta Word Count Tool.

${ }^{5}$ El programa ReCor, diseñado por G. Corpas y M. Seghiri, que permite determinar por primera vez, a posteriori, la representatividad de corpus ha recibido el Premio en Tecnología de la Traducción de España (2007), otorgado por el Observatorio de Tecnologías de la Traducción de la Universidad Europea de Madrid. Para más información, véase Seghiri (2006 y 2015).

' La versión 2.0 difiere de la versión 1.0 en que permite a) seleccionar automáticamente un directorio completo de documentos (en vez de tener que pulsar la tecla Shift como en la versión anterior) y b) seleccionar un número de n-gramas para el cálculo, donde $\mathrm{n} S 1$ y n S 10. Ambas versiones (1.0 y 2.0) generan archivos estadísticos en texto plano (.txt).
}

aleatorio (color azul). De este modo, resulta especialmente llamativo que el subcorpus integrado por los contratos de compraventa de viviendas redactados en Irlanda, a pesar de que la muestra compilada sea de 10 documentos, alcance su representatividad con un solo contrato y con 4.807 palabras). Esto se debe a que la redacción de dichos documentos viene duramente restringida desde el punto de vista legal por la Law Society of Ireland. De este modo, se ha estandarizado el modelo, así como la estructura y contenido de dichos documentos, tal y como se expondrá en el apartado 3.2.1, que dedicaremos al estudio de la estructura de los contratos de compraventa de viviendas en inglés irlandés.

Gracias al programa ReCor y siguiendo los pasos indicados, hemos comprobado que el corpus no solo es representativo a nivel cualitativo, sino también cuantitativo, por lo que estaría listo para ser explotado por cualquier programa de concordancias.

\subsection{Explotación del corpus de contratos de compraventa de viviendas en inglés (variedad irlandesa)}

Finalizado el proceso de compilación del corpus y asegurada su representatividad cualitativa y cuantitativa, el presente apartado está destinado al estudio de la micro- y macroestructura de los contratos de compraventa de viviendas en inglés (en la variedad irlandesa). Para ello, se ha utilizado la herramienta de gestión de corpus comparable gratuita AntConc. Se trata de un programa gratuito y multiplataforma, es uno de los más valorados para la gestión de corpus comparable, pues ofrece un gran número de posibilidades al usuario, como la extracción de listas de palabras y palabras clave, búsquedas de colocaciones, frecuencias absolutas y relativas, entre otras funcionalidades.

\subsubsection{Estructura de los contratos de compraventa de viviendas}

Tal y como se ha señalado anteriormente (vid. apartados 2 y 3.1.2), en lo que a la redacción de contratos de compraventa de viviendas se refiere, en el año 1976 la Law Society of Ireland prescribió una estructura modelo de contrato de compraventa de vivienda para Irlanda, por lo que quedó unificado para agilizar los procesos de compraventa y garantizar la protección de ambas partes. Tras su análisis, se pueden establecer las siguientes partes en el contrato de compraventa de viviendas irlandés:

1. Presentación de las partes que intervienen en el contrato, así como de los abogados de estas, en caso de tenerlos. Asimismo, existe una diferenciación entre venta privada y venta por subasta pública. Además, se mencionan la fecha y lugar de celebración del contrato. 
2. Consentimiento del cónyuge de la parte vendedora conforme a todas las condiciones que se mencionan en el contrato, en el caso de que la vivienda se encuentre sometida al régimen de bienes gananciales. En este punto también se procede a la identificación de los testigos.

3. Identificación fiscal de la parte vendedora y compradora. En este punto se deben recoger los documentos nacionales de identidad de ambas partes, así como el correo electrónico de los abogados de las partes (si los hubiera).

4. Precio de la vivienda. En esta parte se fija el precio de venta, la fecha de cierre de la misma, el depósito de venta y el tipo de interés (porcentaje anual).

5. Datos de los testigos: nombre, domicilio ocupación y firma. Asimismo, deben comparecer dos testigos en total (uno para cada una de las partes).

6. Datos y detalles. Espacio habilitado para que las partes hagan las puntualizaciones que consideren necesarias para la perfección del contrato de compraventa.

7. Descripción de la propiedad, donde se deben indicar, en primer lugar, los datos catastrales de la propiedad y, en segundo lugar, se habilita un espacio en el que se podrán adjuntar los datos relativos a la vivienda que puedan resultar de interés.

8. Condiciones especiales. Apartado en el que las partes indicarán las condiciones especiales de compraventa acordadas, siendo, en todo caso, supletorias. Así, prevalecerán las condiciones generales frente a las particulares y especiales.

9. Condiciones generales de venta: aquí se enumeran, concretamente, las siguientes condiciones:

9.1. Definiciones e interpretación: se describen algunos aspectos con la forma en la que se exponen las condiciones del contrato. De este modo, por ejemplo, se explica el significado y alcance de las expresiones «aceptación», "condiciones» o "fecha de resolución», así como el uso del masculino, plural y singular.

9.2. Venta por subasta: sección en la que se describen las condiciones particulares en el caso de que la venta haya tenido lugar a través de una subasta.

9.3. Venta por acuerdo privado: a diferencia del anterior, se indican las condiciones de venta si esta tiene lugar por un acuerdo privado inter partes.

9.4. Condiciones de común aplicación: venta por subasta o venta por acuerdo privado:

9.4.1. Título: en el que se abordan los siguientes aspectos: en primer lugar, aceptación por ambas partes (comprador y vendedor); en segundo lugar, el comprador declarará haber recibido las copias de los documentos indicados en el contrato; y en tercer lugar, el vendedor deberá haber cancelado las hipotecas y los cargos que afecten a la propiedad en cuestión.
9.4.2. Requisitos posteriores al contrato: donde se le reconoce al comprador el derecho, después de la fecha de venta, a solicitar la inspección de la vivienda.

9.4.3. Título de arrendamiento: cuando la totalidad o parte de la compraventa se celebre en virtud de un arrendamiento; en otras palabras, se conoce esta opción coloquialmente como «alquiler con derecho a compra».

9.4.4. Inscripción de la propiedad: la vivienda objeto del contrato de compraventa deberá estar registrada conforme a lo establecido en el Registration of Title Acts 1891 to $1942 \mathrm{y}$ en el Registration of Title Act 1964. Además, el vendedor debe confirmar, por un lado, que ha entregado al comprador una copia del folio o folios sellados y certificados del Registro de la Propiedad y, por otro lado, que ha indicado en el contrato una descripción elaborada por un tercero competente de acuerdo con lo establecido en Act 1964.

9.4.5. Identidad: el vendedor acepta colaborar con el comprador en el caso de que desee examinar datos relativos a los indicados en el listado de documentos. Así, deberá comprometerse a proporcionar al comprador la información que posea en relación con la propiedad; sin embargo, no se le exigirá a este que detalle información muy concreta, como los límites, cercas, zanjas o muros.

9.4.6. Vendedor como persona jurídica: cuando la parte vendedora sea una compañía, corporación, asociación u otra entidad similar, se deberá indicar este hecho en el apartado relativo a las condiciones especiales del contrato.

9.4.7. Derechos, responsabilidades y condiciones a los que está sujeta la propiedad: en este apartado el vendedor confirma que ha revelado, antes de la fecha de venta, en el apartado de condiciones especiales, todas las servidumbres, derechos, reservas, excepciones, arrendamientos, impuestos y otras responsabilidades que afectan a la propiedad objeto de la compraventa.

9.4.8. Búsquedas: el vendedor confirma que ha proporcionado al comprador, antes de la fecha de venta, todos los datos pertenecientes para la realización de cualquier búsqueda en el registro de la propiedad.

9.4.9. Garantía: aquellas cláusulas accesorias que se suscriben para asegurar el cumplimiento de un contrato principal.

9.4.10. Posesión vacante: si el vendedor incumple cualquiera de las condiciones y disposiciones recogidas en el contrato, el comprador tendrá derecho a la posesión vacante de la propiedad sujeta.

9.4.11. Arrendamientos: cuando la propiedad en cuestión se vende sujeta a cualquier arrendamiento, el vendedor debe entregar una copia contractual del mismo. Además, a menos que en las condiciones es- 
peciales se establezca lo contrario, el arrendamiento mencionado seguirá en vigor.

9.4.12. Término e interés: la venta se completará en la fecha de cierre o antes. Si por incumplimiento por parte del vendedor la venta no se hubiese realizado antes de la fecha de cierre, este deberá pagar una compensación al comprador.

9.4.13. Fraccionamiento y reparto de frutos: el comprador tendrá derecho a la posesión vacante de la propiedad sujeta o, según sea el caso, a las ganancias y gastos a partir de la fecha de venta.

9.4.14. Registro obligatorio: si la propiedad no se encuentra registrada, el vendedor estará obligado a elevar dicho registro antes de la fecha de finalización.

9.4.15. Firmado como fideicomisario o representante legal: un comprador que firme como fideicomisario o representante legal (o figura similar), sin especificar la identidad de la parte principal, deberá ser personalmente responsable de completar la venta y cumplir todas las estipulaciones adicionales que figuren en las condiciones, a menos que el vendedor indique el nombre del comprador.

9.4.16. Incumplimiento pago de depósito: en el caso de que el comprador no haya pagado en su totalidad el depósito anteriormente especificado, incurrirá en incumplimiento del contrato y autorizará al vendedor a disolver la venta o demandar al comprador.

9.4.17. Diferencias y errores: el término «error» se refiere a cualquier omisión, no declaración, diferencia, inexactitud, declaración o representación errónea manifestada por el vendedor; así, el comprador tendrá derecho a ser compensado económicamente por el vendedor por cualquier pérdida sufrida por dicho «error».

9.4.18. Documentos de otros títulos relacionados con la propiedad: el vendedor $u$ otras personas con derecho a la posesión de estos conservarán los documentos relacionados con el objeto de venta; por ejemplo, cuando la propiedad esté sujeta a venta en lotes, la parte vendedora podrá poseer los documentos relacionados con dicha venta hasta la finalización de las ventas de todos los lotes.

9.4.19. Notificaciones: antes de la fecha de venta, cualquier hecho relacionado con el bien inmueble ha de ser notificado al comprador en la forma en la que el comprador lo haya autorizado.

9.4.20. Desarrollo: el vendedor, antes de la venta de la vivienda, debe poner a disposición del comprador copias de todos los documentos relacionados con los permisos de planificación o certificados de seguridad contra incendios, entre otros.

9.4.21. Resolución: finalizada la venta, de acuerdo con las disposiciones del contrato, el compra- dor tendrá derecho a la devolución del depósito, salvo en aquellos casos en los que se haya perdido legalmente el derecho a este.

9.4.22. Notificación de la resolución de la venta: si la venta no se perfecciona antes de la fecha prevista, cualquiera de las partes podrá instar a su resolución. También tendrá derecho a completar dicha venta en un plazo de veintiocho días a contar a partir de dicho requerimiento.

9.4.23. Pérdida de depósito y reventa: si el comprador incumple alguna de las condiciones de venta estipuladas, podrá perder el depósito aportado.

9.4.24. Daños por defecto: sin perjuicio de cualquier norma que establezca lo contrario, el incumplimiento por parte del vendedor de alguna de las condiciones estipuladas en el contrato dará lugar a una indemnización por daños y perjuicios en favor del comprador.

9.4.25. Riesgos: el vendedor será responsable de cualquier pérdida o daño que se ocasione a la propiedad hasta la fecha de venta, pero dicha responsabilidad no deberá exceder el monto total del precio de compra; sin embargo, no se aplicará en caso de deterioro poco importante debido al desgaste razonable por el uso y disfrute adecuado.

9.4.26. Bienes muebles: el vendedor garantizará que, al finalizar la venta, todos los bienes adquiridos por el comprador están libres de cargas y no sujetos a ningún contrato de arrendamiento, de cualquier tipo, o venta a crédito.

9.4.27. Inspección: el vendedor deberá facilitar las solicitudes de inspección que desee realizar el comprador en relación con el bien inmueble en cuestión.

9.4.28. No concentración: sin perjuicio de la garantía de la propiedad entregada al comprador, todas las garantías y disposiciones a perdurar a la finalización del cierre de venta y todas aquellas que no hayan sido aplicadas y que puedan continuar o surtir efecto después del cierre permanecerán en pleno vigor y derecho.

9.4.29. Notificaciones y avisos: a menos que se indique expresamente lo contrario, cualquier notificación se hará por escrito.

9.4.30. Límites: donde se establece el método para la consideración de plazos de cara a la realización de cualquier trámite; así, se hace una breve descripción del cómputo de los plazos a día, mes y año.

9.4.31. Resolución de disputas: todas las diferencias y disputas entre el vendedor y el comprador, salvo acuerdo en contrario de las partes, se someterán en primera instancia a mediación.

Una vez descritas las partes relativas al contrato de compraventa de viviendas de Irlanda, se procederá a la implementación de la plantilla de redacción. 


\subsubsection{Implementación de una plantilla de redacción de contratos de compraventa de viviendas}

Para la implementación de una plantilla de redacción de contratos de compraventa de viviendas en inglés, en su variedad diatópica de Irlanda, es necesario volver a subrayar el constreñimiento legal en su estructura prescrito por la Law Society of Ireland, que no permite que pueda ser modificada. Así, con esta estructura en mente y con objeto de ofrecer una herramienta de fácil manejo para el usuario interesado, se ha implementado la plantilla de redacción en Excel (.xIs). Gracias al estudio de la muestra que constituye nuestro corpus, podemos presentar, a modo resumen, una tabla que recoge la estructura y principal forma de redacción de cada parte del contrato de compraventa irlandés (cfr. Tabla 3).

\begin{tabular}{|c|}
\hline LAW SOCIETY CONDITIONS OF SALE \\
\hline SPOUSAL*CIVIL PARTNER CONSENT \\
\hline MEMORANDUN AGREEMENT \\
\hline PARTICULARS AND TENURE \\
\hline DOCUMENTS SCHEDULE \\
\hline GENERAL CONDITIONS OF SALE \\
\hline DEFINITIONS AND INTERPRETATION \\
\hline AUCTION \\
\hline PRIVATE TREATY SALE \\
\hline REOUISITIONS \\
\hline POST-CONTRACT REOUISITONSPOST-CONTRACT \\
\hline LOST-CONTRACT REQUISITIONS \\
\hline REGISTERED LAND \\
\hline IDENTITY \\
\hline FOREIGN VENDOR \\
\hline RIGHTS - LIABILITIES - CONDITION OF SUBJECT PROPERTY \\
\hline SEARCHES \\
\hline ASSURANCE \\
\hline
\end{tabular}

Tabla 3. Estructura y contenido del contrato de compraventa de viviendas de Irlanda.

Por su parte, la plantilla de contrato de compraventa de viviendas en inglés irlandés, con este contenido, se ha implementado en formato .xls (cfr. Figura 7).

Como resultado de la metodología ilustrada en la presente sección, hemos obtenido una plantilla de contrato de compraventa de viviendas en inglés irlandés. La citada plantilla se encuentra disponible, para su consulta, también en línea?

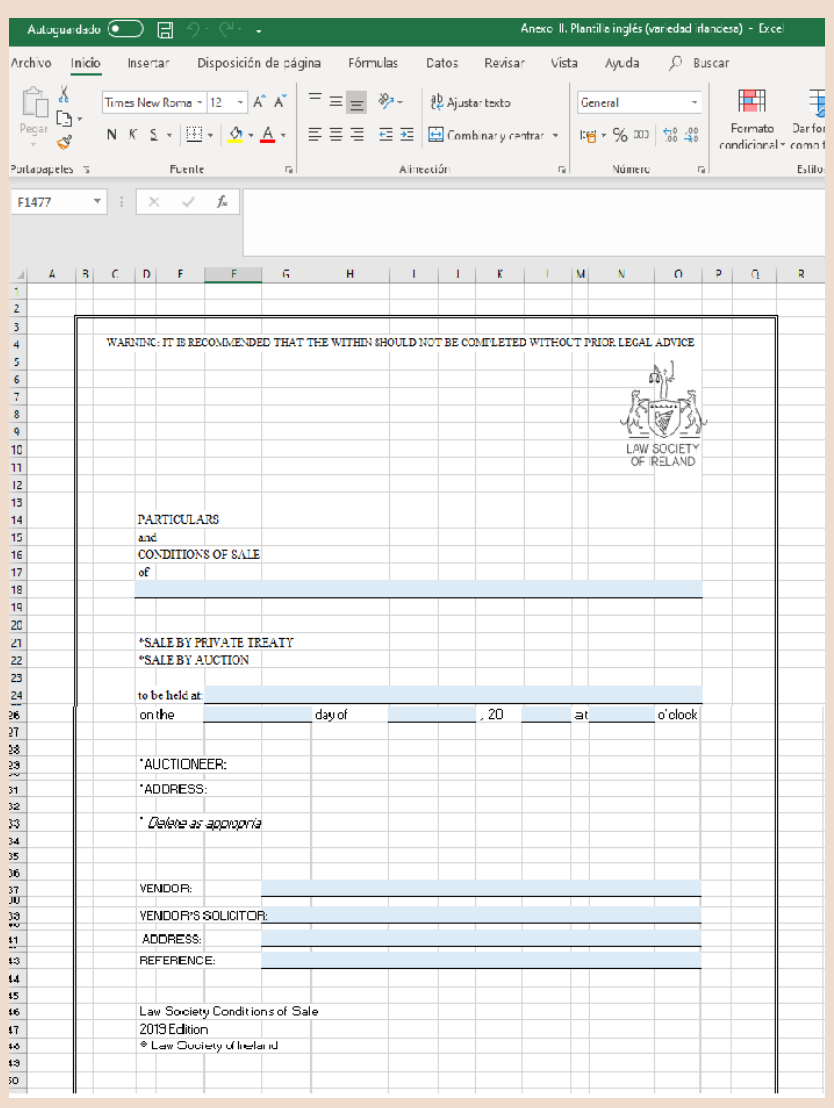

Figura 7. Plantilla en formato .xls de contratos de compraventa de viviendas en inglés (variedad irlandesa).

\section{Conclusiones}

Los corpus comparables son un recurso de gran utilidad para el desempeño de la traducción, especialmente para el discurso especializado y, más concretamente, el jurídico, por el carácter estereotipado que lo define. Así, en el presente trabajo se ha ilustrado el proceso de implementación de una plantilla en inglés (variedad irlandesa) de contratos de compraventa de viviendas, capaz de semiautomatizar el proceso de redacción de dichos documentos. Asimismo, se ha identificado la legislación aplicable a la compraventa de viviendas en Irlanda, hecho que ha incidido directamente en la redacción de estos contratos en tanto que viene limitada por la Law Society of Ireland. A partir del corpus de contratos de compraventa de viviendas compilado a tal efecto, a su vez, hemos podido extraer las estructuras recurrentes para la redacción de cada parte de obligado contenido en el contrato, en función de la citada ley irlandesa. Finalmente, esta información macro-y microestructural se ha volcado en un documento en Excel que se ha puesto gratuitamente a disposición de cualquier usuario que lo necesite a través de la red Internet. 


\section{Agradecimientos}

La metodología de investigación aquí descrita ha recibido el Premio George Campbell del Aula María Zambrano de Estudios Transatlánticos, de la Universidad de Málaga (2019). Asimismo, el presente trabajo se enmarca en el seno de la red docente de excelencia TACTRAD (Ref. 719/2018) de la Universidad de Málaga, así como en el marco de los proyectos VIP (Ref. FFI2016-75831-P), Training network on language technologies for interpreters (Ref. EUIN2017-87746), aplicación de técnicas avanzadas de PLN al ámbito de las tecnologías de la traducción y la interpretación (Ref. EQC2018-004572-P), MI4ALL (AndalucíaTech), introducción al mercado profesional de la Tel (UMA 2020), POSTrad III (Ref. PIE 102, UVa), TRIAJE (Ref. UMA18-FEDERJA-067) y PROFETA (Ref. PIE19-033, UMA).

\section{Fuentes ybibliografía}

\section{Referencias bibliográficas}

Arce Romeral, L., y Seghiri, M. (2018): «Determinación de la representatividad cualitativa y cuantitativa de un corpus virtual de contratos de compraventa de viviendas (inglés-español)», en García Peinado, M. A., y Ahumada Lara, E. (eds.): Traducción literaria y discursos traductológicos especializados, pp. 309-330. Ámsterdam: Peter Lang (ISBN: 978-3-631-74680-6).

-(2019): «Diseño de plantillas de redacción y traducción al inglés (variedades británica y estadounidense) de contratos de compraventa de viviendas basadas en corpus», en Seghiri, M. (ed.): La lingüística de corpus aplicada al desarrollo de la competencia tecnológica en los estudios de traducción e interpretación y la enseñanza de segundas lenguas, pp. 69-106. Ámsterdam: Peter Lang (ISBN: 978-3-631-74122-1).

-(2020): «Implementación de plantillas de redacción de contratos de compraventa de viviendas en español: variedades peninsular y argentina», en García, M. A., y Torralbo, J. de Dios (eds.): La práctica de la traducción especializada jurídica (en prensa). Ámsterdam: Peter Lang.

Corpas Pastor, G. (2001): «Compilación de un corpus ad hoc para la enseñanza de la traducción inversa especializada», en TRANS. Revista de Traductología, pp. 155184.

-(2008): Investigar con corpus en traducción: los retos de un nuevo paradigma. Fráncfort del Meno: Peter Lang.

EAGLES (1996): Preliminary Recommendations on Corpus Typology, documento EAGLES (Expert Advisory Group on Language Engineering) EAGTCWG-CTYP/P.

Seghiri, M. (2006): Compilación de un corpus trilingüe de seguros turísticos (español-inglés-italiano): aspectos de evaluación, catalogación, diseño y representatividad. (Tesis doctoral, Universidad de Málaga). Recuperado de https://riuma.uma.es/xmlui/handle/10630/2715

-(2011): «Metodología protocolizada de compilación de un corpus de seguros de viajes: aspectos de diseño y representatividad», en Revista de Lingüística Teórica y Aplicada, 49 (2), pp. 13-30. Recuperado de http://dx. doi.org/10.4067/S0718-48832011000200002

-(2012): «Creating Electronic Corpora: Design, Compilation Protocol and representativeness», en Parra, E.; García, M. A.; y Classen, A. (eds.): Aspects of Literary Translation: Building Linguistic and Cultural Bridge in Past and Present, pp. 373-382. Tubinga: Narr Verlang.

-(2014): «Too big or not too big: Establishing the minimum size for a legal ad hoc corpus», en Hermes Journal, 53, pp. 85-98. Recuperado de https://tidsskrift.dk/her/article/ view/20981

-(2015): «Determinación de la representatividad cuantitativa de un corpus ad hoc bilingüe (inglés-español) de manuales de instrucciones generales de lectores electrónicos / Establishing the quantitative representativiness of an E-Reader User's Guide ad hoc corpus (English-Spanish)», en Sánchez Nieto, M. T. (ed.): Corpus-based Translation and Interpreting Studies: From description to application, pp. 125-146. Berlín: Frank \& Timme.

-(2017a): «Metodología de elaboración de un glosario bilingüe y bidireccional (inglés-español/español-inglés) basado en corpus para la traducción de manuales de instrucciones de televisores. Creating a bilingual and bidirectional glossary (English-Spanish/Spanish-English) based on corpus for the translation of TV user manuals», en Babel, 63 (1), pp. 43-64. John Benjamins Publishing Company.

-(2017b): «Corpus e interpretación biosanitaria: extracción terminológica basada en bitextos del campo de la neurología para la fase documental del intérprete», en Panacea, 18 (46), pp. 123-132.

Sinclair, J. (1991): Corpus Concordance Collocation. Oxford: Oxford University Press.

\section{Informes, estadísticas y artículos de prensa}

Aranda, J. L. (2018): «Los extranjeros compran más casas que nunca en España: quiénes son y dónde invierten», en El País, 15 de noviembre. Recuperado de https://elpais.com/economia/2018/11/14/vivienda/ 1542193768 934754.html

INE (2001): Censo de población y viviendas. Recuperado de http://www.ine.es/censo2001/censo2001.htm

-(2011): Censo de población y viviendas. Recuperado de https://www.ine.es/censos2011 datos/cen 11 datos inicio.htm

-(2018): Estadística de movimientos turísticos en fronteras (FRONTUR). Recuperado de http://www.ine.es/daco/ daco42/frontur/frontur0219.pdf

Ministerio de Trabajo, Migraciones y Seguridad Social del Gobierno de España (2016): Datos sociológicos de la emigración española en Irlanda. Recuperado de http:// www.mitramiss.gob.es/es/mundo/consejerias/reino Unido/emigracion/contenidos/datosirlanda.htm

Thomson Reuters (2013): «Los extranjeros ya compran 20 de cada 100 viviendas que se venden en España», en El Confidencial, 15 de octubre. Recuperado de https:// www.elconfidencial.com/vivienda/2013-10-15/losextranjeros-ya-compran-20-de-cada-100-viviendasque-se-venden-en-espana 41404/

World Tourism Organization, UNWTO (2019): Panorama OMT del turismo internacional. Recuperado de https:// www.e-unwto.org/doi/pdf/10.18111/9789284421237 


\section{Referencias legislativas}

Constitution of Ireland, 1937. Electronic Irish Statute Book (elSB), 1 de julio de 1937. Recuperado de http://www. irishstatutebook.ie/eli/cons/en/htm/\#a\%20rticle43

Family Home Protection Act, 1976. Electronic Irish Statute Book (elSB), 12 de julio de 1976, núm. 27, pp. 1-9. Recuperado de http://www.irishstatutebook.ie/eli/1976/ act/27/enacted/en/html

Irish Land (Provision for Sailors and Soldiers) Act 1919. Electronic Irish Statute Book (elSB), 23 de diciembre de 1919, núm. 82, pp. 1-6. Recuperado de http://www. irishstatutebook.ie/eli/1919/act/82/enacted/en/html

Land (Purchase) Act, 1903. Electronic Irish Statute Book (elSB), 14 de agosto de 1903, núm. 37, pp. 1-45. Recuperado de http://www.irishstatutebook.ie/eli/1903/ act/37/enacted/en/print.html

Land Act, 1923. Electronic Irish Statute Book (elSB), 9 de agosto de 1923, núm.42, pp. 1-40. Recuperado de http:// www.irishstatutebook.ie/eli/isbc/1923.html\#a42 1923

Land and Conveyancing Law Reform Act, 2009. Electronic Irish Statute Book (elSB), 21 de julio de 2009, núm. 27, pp. 1-143. Recuperado de http://www.irishstatutebook. ie/eli/2009/act/27/enacted/en/html

Land and Conveyancing Law Reform Act, 2013. Electronic Irish Statute Book (elSB), 23 de julio de 2013, núm. 30, pp. 1-6. Recuperado de http://www.irishstatutebook.ie/ eli/2013/act/30/enacted/en/html

Land and Conveyancing Law Reform Act, 2019. Electronic Irish Statute Book (elSB), 10 de julio de 2019, núm. 22, pp. 1-6. Recuperado de http://www.irishstatutebook.ie/ eli/2019/act/22/section/1/enacted/en/html

Land Purchase Act, 1887. Electronic Irish Statute Book (elSB), 23 de agosto de 1887, núm. 33, pp. 1-25. Recuperado de http://www.irishstatutebook.ie/eli/1887/ act/33/enacted/en/print.html

Land Registration (Fees) Order, 1966. Electronic Irish Statute Book (elSB), 1 de abril de 1966, núm. 57, pp. 1-30. Recuperado de http://www.irishstatutebook.ie/eli/1966/ si/57/made/en/print

Land Registration (Fees) Order, 1974. Electronic Irish Statute Book (elSB), 30 de octubre de 1974, núm. 276, pp. 1-15. Recuperado de http://www.irishstatutebook.ie/eli/1974/ si/315/made/en/print

Land Registration (Fees) Order, 1978. Electronic Irish Statute Book (elSB), 8 de marzo de 1978, núm. 40, pp. 1-21. Recuperado de http://www.irishstatutebook.ie/eli/1978/ si/40/made/en/print

Land Registration (Fees) Order, 1980. Electronic Irish Statute Book (elSB), 1 de agosto de 1980, núm. 255, pp. 1-58. Recuperado de http://www.irishstatutebook.ie/eli/1980/ si/255/made/en/print

Land Registration (Fees) Order, 1981. Electronic Irish Statute Book (elSB), 1 de diciembre de 1981, núm. 255, pp. 1-15. Recuperado de http://www.irishstatutebook. ie/eli/1981/si/370/made/en/print

Land Registration (Fees) Order, 1983. Electronic Irish Statute Book (elSB), 1 de marzo de 1983, núm. 16, pp. 1-12. Recuperado de http://www.legislation.ie/eli/1983/si/388/ made/en/print

Land Registration (Fees) Order, 1987. Electronic Irish Statute Book (elSB), 1 de febrero de 1987, núm. 16, pp. 1-7. Recuperado de http://www.irishstatutebook.ie/eli/1987/ si/249/made/en/print
Land Registration (Fees) Order, 1991. Electronic Irish Statute Book (eISB), 1 de abril de 1992, núm. 16, pp. 1-18. Recuperado de http://www.irishstatutebook.ie/eli/1991/ si/363/made/en/print

Land Registration (Fees) Order, 1999. Electronic Irish Statute Book (elSB), 1 de mayo de 2002, núm. 16, pp. 1-18. Recuperado de http://www.irishstatutebook.ie/eli/1999/ si/343/made/en/print

Land Registration Rules, 1975. Electronic Irish Statute Book (elSB), 16 de octubre de 1975, núm. 246, pp. 1-9. Recuperado de http://www.irishstatutebook.ie/eli/1975/ si/246/made/en/print

Land Registration Rules, 1977. Electronic Irish Statute Book (elSB), 24 de febrero de 1977, núm. 89, pp. 1-16. Recuperado de http://www.irishstatutebook.ie/eli/1977/ si/89/made/en/print

Land Registration Rules, 1986. Electronic Irish Statute Book (elSB), 1 de octubre de 1986, núm. 310, pp. 1-30. Recuperado de http://www.irishstatutebook.ie/eli/1986/ si/310/made/en/print

Land Registration Rules, 2000. Electronic Irish Statute Book (elSB), 1 de junio de 2002, núm. 175, pp. 1-63. Recuperado de http://www.irishstatutebook.ie/eli/2000/si/175/ made/en/print

Land Registration Rules, 2005. Electronic Irish Statute Book (elSB), 1 de enero de 2006, núm. 643, pp. 1-68. Recuperado de http://www.irishstatutebook.ie/eli/2005/si/643/ made/en/print

Land Registration Rules, 2006. Electronic Irish Statute Book (elSB), 4 de noviembre de 2006, núm. 558, pp. 1-12. Recuperado de http://www.irishstatutebook.ie/eli/2006/si/558/ made/en/print

Land Registration Rules, 2007. Electronic Irish Statute Book (elSB), 7 de agosto de 2007, núm. 568, pp. 1-10. Recuperado de http://www.irishstatutebook.ie/eli/2007/si/ 568/made/en/print

Land Registration Rules, 2009. Electronic Irish Statute Book (elSB), 1 de noviembre de 2009, núm. 349, pp. 1-28. Recuperado de http://www.irishstatutebook.ie/eli/2009/si/ 349/made/en/print

Land Registration Rules, 2011. Electronic Irish Statute Book (elSB), 8 de noviembre de 2011, núm. 559, pp. 1-14. Recuperado de http://www.irishstatutebook.ie/eli/2011/si/ 559/made/en/print

Land Registration Rules, 2012. Electronic Irish Statute Book (elSB), 1 de febrero de 2013, núm. 483, pp. 1-223. Recuperado de http://www.irishstatutebook.ie/eli/2012/si/ 483/made/en/print

Land Registration Rules, 2013. Electronic Irish Statute Book (elSB), 15 de octubre de 2013, núm. 389, pp. 1-30. Recuperado de http://www.irishstatutebook.ie/eli/2013/si/ 389/made/en/print

Purchase of Land (Ireland) Act 1885. Electronic Irish Statute Book (elSB), 14 de agosto de 1885, núm. 73, pp. 1-12. Recuperado de http://www.irishstatutebook. ie/eli/1885/act/73/enacted/en/print.htm/

Registration of Deeds and Title Act, 2006. Electronic Irish Statute Book (elSB), 7 de mayo de 2006, núm. 12, pp. 1-41. Recuperado de http://www.irishstatutebook. ie/eli/2006/act/12/enacted/en/print.html

Registration of Title Act, 1964. Electronic Irish Statute Book (elSB), 4 de julio de 1964, núm. 16, pp. 1-57. Recuperado de http:// www.irishstatutebook.ie/eli/1964/act/16/enacted/en/html 
Succession Act, 1965. Electronic Irish Statute Book (elSB), 22 de diciembre de 1965, núm. 27, pp. 1-68. Recuperado de http://www.irishstatutebook.ie/eli/1965/act/27/ enacted/en/html

\section{Programas informáticos y recursos en la web}

Anthony's, L. (2019): AntConc (Windows 3.5.8) [software]. Recuperado de https://www.scribbr.es/normas-apa/ejemplosde-las-normas-apa/ejemplo-del-estilo-apa-software/

GetBot (3.04) [software] (2019): Recuperado de http:// www.getbot.com/

GNUWget (en línea) [software] (2017). Recuperado de https://www.gnu.org/software/wget/wget.html

Law Society of Ireland (2019): Recuperado de https://www. lawsociety.iel
Law Society of Ireland (2019): Conditions of Sale 2019 Edition. Recuperado de https://www.lawsociety.ie/ globalassets/documents/committees/conveyancing/ precedents/2019/2019-conditions-of-sale.pdf

PDF Converter (en línea) [software] (2019): Recuperado de https://www. freepdfconvert.com/es

PDF Word (en línea) [software] (2019): Recuperado de https://www.pdftoword.com/es/

Seghiri, M., y Corpas Pastor, G. (2007): ReCor (2.0) [software]. Recuperado de https://www.uma.es/hum892/Publica ciones/Corpas Seghiri 2007g.pdf

Vlex (2019): Vlex Ireland. Recuperado de https://ie.vlex. $\mathrm{com} /$

WordCount Tool (en línea) [software] (2019): Recuperado de http://wordcountool.com 